\title{
Transverse load and orientation measurement with multicore fiber Bragg gratings
}

\author{
Manuel Silva-López, William N. MacPherson, Cheng Li, Andrew J. Moore, \\ James S. Barton, Julian D. C. Jones, Donghui Zhao, Lin Zhang, and lan Bennion
}

\begin{abstract}
We demonstrate the sensitivity of Bragg gratings in a multicore fiber to transverse load. The Bragg peaks are split because of stress-induced birefringence, the magnitude of which depends upon the load and grating position relative to the load axis. Experiments show that a set of gratings in a four-core fiber can measure a load axis angle to $\pm 5^{\circ}$ and a load magnitude to $\pm 15 \mathrm{~N} \mathrm{~m}^{-1}$ up to $2500 \mathrm{~N} \mathrm{~m}^{-1}$. We consider alternative designs of multicore fiber for optimal load sensing and compare experimental and modeled data. () 2005 Optical Society of America
\end{abstract}

OCIS codes: $\quad 060.2370,260.1440,230.1480$.

\section{Introduction}

Fiber Bragg gratings (FBGs) are now well established in many applications requiring longitudinal strain measurement. The additional possibility of transverse strain measurement is introduced by the action of a transverse load when the refractive-index profile of a fiber is modified to induce birefringence. ${ }^{1,2}$ Fiber sensors capable of measuring transverse loads can open up new possibilities in multiaxis load sensing, for example, to investigate the strain field in composite materials ${ }^{3,4}$ or as all-fiber alternatives to load cells for engineering and process applications requiring compact sensors for hazardous environments.

Birefringence induced in a conventional singlemode fiber subject to transverse loading will cause the spectrum of a simple uniform FBG to split into two peaks. To determine the angular orientation of the load, it is necessary to make two independent measurements of the birefringence-induced wavelength shift at known orientations within the fiber. A convenient way to do this is to use FBGs written in highly birefringent (hi-bi) fiber. 5 The Bragg wave-

M. Silva-López, W. N. MacPherson (w.n.macpherson@hw.ac.uk), C. Li, A. J. Moore, J. S. Barton, and J. D. C. Jones are with the Applied Optics and Photonics Group, School of Engineering and Physical Sciences, Heriot-Watt University, Edinburgh EH14 4AS, UK. D. Zhao, L. Zhang, and I. Bennion are with the Photonics Research Group, Electronic Engineering and Computer Science Division, Aston University, Birmingham B4 7ET, UK.

Received 25 April 2005; accepted 6 July 2005.

0003-6935/05/326890-08\$15.00/0

(C) 2005 Optical Society of America length shifts corresponding to the two orthogonal polarization modes are approximately linear functions of the transverse load. More recently, the principle of temperature compensation in transverse strain measurement in hi-bi fiber has been demonstrated by using two superimposed gratings of slightly different Bragg wavelengths. ${ }^{6} \mathrm{~A}$ change in temperature or in longitudinal strain produces a common-mode shift in both FBGs, while the transverse strain sensitivities of the two polarization modes differ. Long-period gratings exhibit higher sensitivity ${ }^{7}$ to transverse strain than do FBGs, since the cladding modes are more strongly affected by the refractive-index changes induced by the loading than are the core modes, but interpretation of their response is less straightforward than for FBGs.

In this paper we investigate the relative birefringence of the cores in a multicore fiber (MCF) as a function of load and its orientation. In this application, the MCF has the advantage of several cores that are not located at the center of the fiber cross section. Hence FBGs written in these cores will sample the transverse strain field at a number of points. The transverse strain is greatest close to the edge of the cladding along the diameter aligned with the load axis and is least at points furthest from the load axis. The difference in Bragg peak splitting between pairs of gratings can be used to measure the magnitude and orientation of the applied transverse load. We have previously reported ${ }^{8}$ this technique in a fourcore MCF, in which we observed a linear increase in birefringence peak splitting with an applied load. In this paper we investigate the orientation sensitivity of a MCF and compare it with the response calculated 


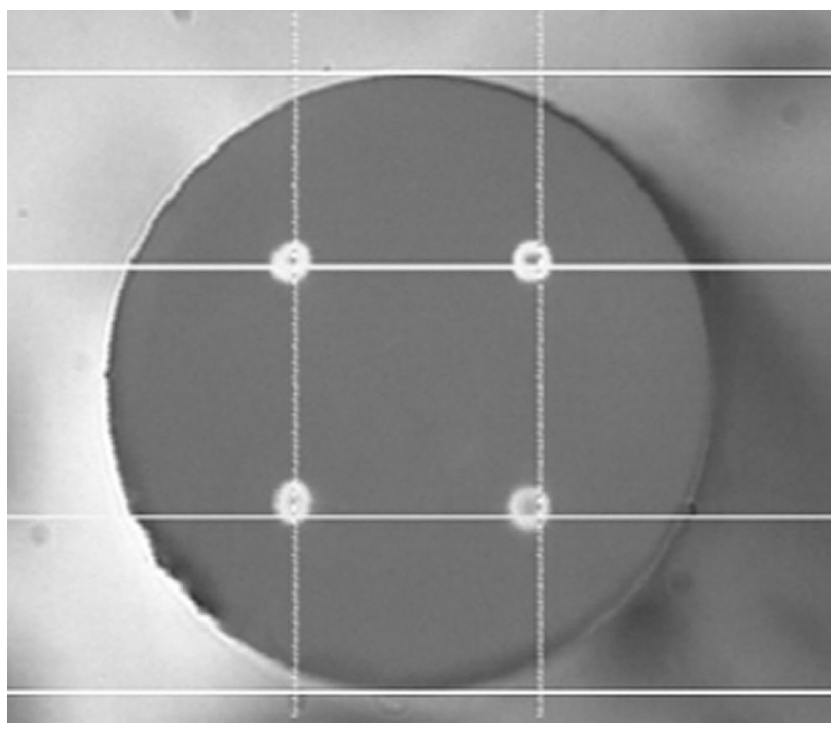

Fig. 1. MCF cross section. The core spacing is $50 \mu \mathrm{m}$.

from the elastic analysis of a transversely loaded fiber.

\section{Transverse Loading Experiments}

\section{A. Multicore Fiber}

MCF has been reported ${ }^{9}$ as a possible technique for multichannel communications. Our interest in MCF arises from its potential application to sensing. The MCF used in this experiment was fabricated from a standard fused-silica preform drilled to accept fourboron-codoped germanosilicate ${ }^{10}$ rods that form the cores when drawn into the fiber. The rods were arranged at the vertices of a square, resulting in four cores with a separation of $50 \mu \mathrm{m}$ when the preform was drawn to a fiber with a cladding diameter of $125 \mu \mathrm{m}$, as shown in Fig. 1. Each core was single mode at $1550 \mathrm{~nm}$ and separated sufficiently from its neighbors to prevent any observable cross talk. The circular cross section was particularly important to facilitate fiber handling, and we observed that higher-quality cleaves were obtained using this fiber in contrast to other (noncircular) MCFs ${ }^{11}$ that we tested previously. This has allowed us to make lowloss $(\approx 1 \mathrm{~dB})$ splices between lengths of $\mathrm{MCF}$ using a fusion splicer with a fiber rotation and alignment facility designed to connect high-birefringence fibers.

Application of a MCF in practical situations requires the technology to be developed to the point at which it is as simple and convenient to use as a conventional optical fiber. We have previously described a fan-out ${ }^{8}$ that interfaces the MCF to standard single-core fibers, thus enabling interrogation optics and systems to be constructed using commercially available components. It is also necessary to consider connections between lengths of MCF, and for convenience it is desirable to be able to use a MCF terminated with fiber connectors, rather than fusion splice each connection. To achieve low-loss coupling

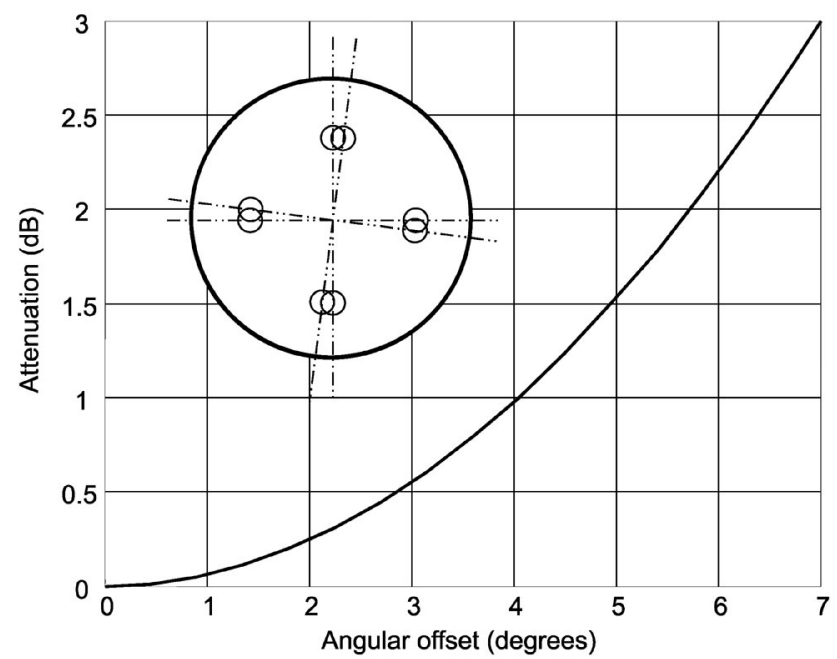

Fig. 2. Calculated attenuation due to angular misalignment between two MCFs.

between fibers it is critical that the four cores in the fiber connector are carefully aligned. To investigate the tolerances required, a simulation of the losses due to core misalignment was made using the Gaussian approximation for single-mode circular waveguides. Figure 2 shows the calculated attenuation in each core as a function of angular misalignment. From this we conclude that even a few degrees of offset are enough for appreciable losses, and to achieve coupling losses lower than $1 \mathrm{~dB}$ requires alignment better than $\pm 4^{\circ}$.

The fabrication of the MCF connectors was similar to the procedure used to align connectors for polarization-maintaining hi-bi fibers. The MCF was glued into a keyed connector mounted in a holder such that the orientation of the connector was well controlled. The end of the fiber protruding from the connector was imaged with a microscope. Using alignment marks on the microscope it was possible to adjust the rotation of the fiber before the adhesive cured. In this way it is possible to achieve angular alignment of the cores to the desired position relative to the connector keyway with an accuracy better than $5^{\circ}$. Once the adhesive cured, the fiber end face was polished level with the connector end face. Figure 3 shows the end view of the connector after polishing, and the inset shows the final schematic configuration of the MCF connector keyed with respect to the cores. Using a standard bulkhead adapter to align two MCFs, connector losses of less than $1 \mathrm{~dB}$ have been obtained with cross talk between the cores below $-48 \mathrm{~dB}$.

\section{B. Bragg Gratings in Multicore Fibers}

The intrinsic photosensitivity of this MCF permitted UV inscription of FBGs in the cores without hydrogenation treatment of the fiber. We wrote highefficiency FBGs in the MCF using the standard phase mask scanning technique and a frequency-doubled Ar laser with a $95 \mathrm{~mW}$ output at $244 \mathrm{~nm}$, with a 


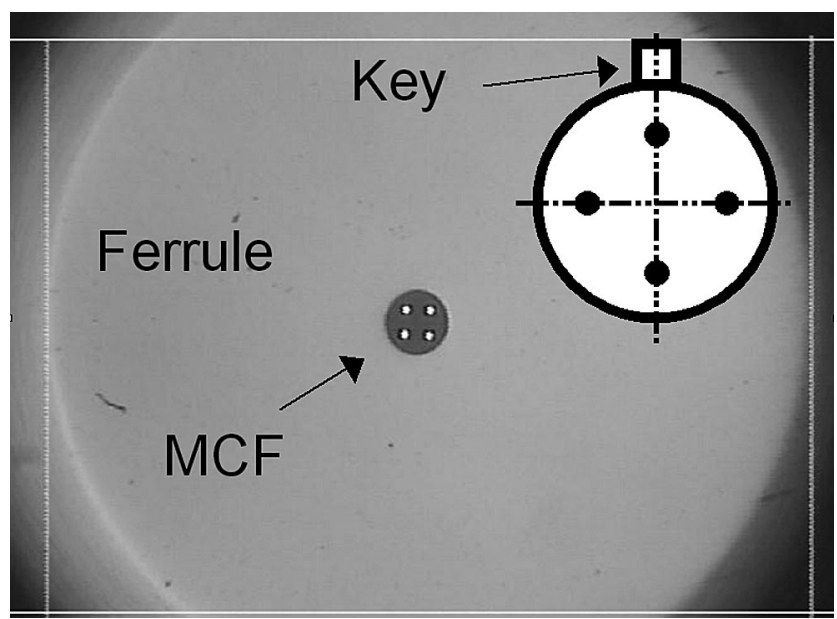

Fig. 3. Front face of the MCF in a fiber connector. Inset shows the position of the cores with respect to the connector keyway.

typical scan speed of $0.07 \mathrm{~mm} \mathrm{~s}^{-1}$ over a $10 \mathrm{~mm}$ length of fiber. To write gratings selectively in the cores, we mounted the fiber on a rotation stage to accommodate the setting of the fiber orientation. A double-exposure method was found to be effective to ensure the inscription to all four cores. This involved a second UV exposure to the fiber after rotation of $180^{\circ}$ following the first exposure. As can be seen from the first row of Fig. 4, the FBG strengths $(\sim 50 \%$ reflectivity) in two cores may differ slightly because of the small variations in UV intensities experienced by the four cores, which we believe can be equalized by further control of the fabrication procedure.

The MCF under test contained FBGs in four cores at the same axial position and was interrogated using the configuration shown in Fig. 5. The test MCF (i.e.,
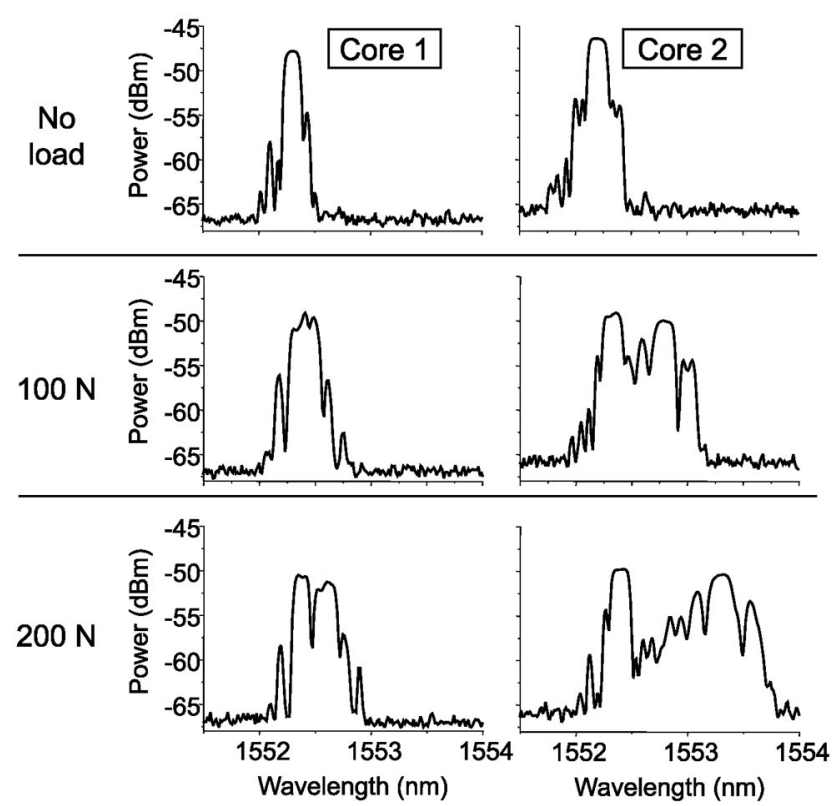

Fig. 4. Reflected FBG spectra from two MCF cores showing peak splitting under an increasing transverse load.

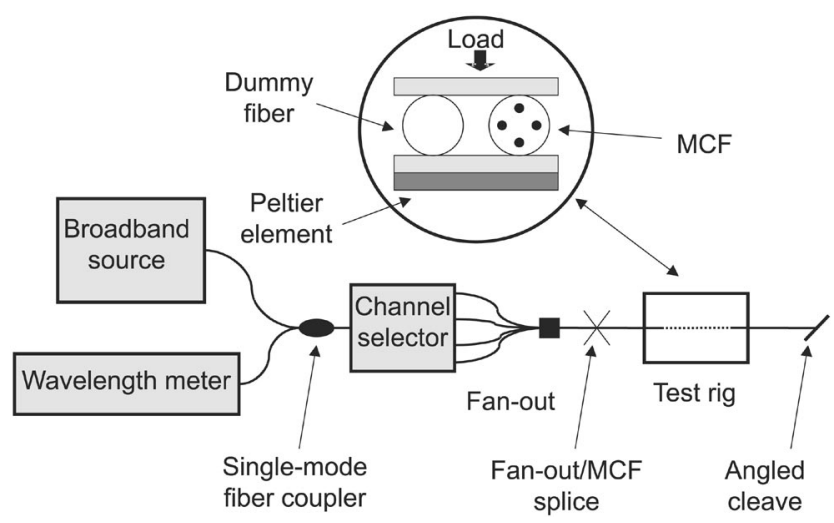

Fig. 5. Interrogation system with selectable addressing of the four MCF cores.

the sensing fiber) was fusion spliced onto a length of MCF that formed part of the fan-out assembly ${ }^{8}$ that interfaced four standard SMF-28 input fibers to one MCF, such that each input addressed only one of the MCF cores. The four input fibers could be selected individually using a fiber switch (channel selector), thus enabling the FBG reflection spectrum from each of the four cores to be interrogated sequentially. The interrogation system comprised a broadband source with a wavelength range of $1530-1560 \mathrm{~nm}$ and an output power of $\sim 11 \mathrm{dBm}$ and a wavelength meter with $14 \mathrm{pm}$ resolution. The fiber was held between two flat polished aluminum plates such that it was parallel to a second dummy fiber, both of which were stripped of the outer buffer; this configuration ensured that the applied load was distributed uniformly by constraining the two plates to remain parallel under loading. The load was applied by adding masses to the top aluminum plate via a system of loading plates to ensure that the load was evenly distributed. The fiber was coated with thermal oil to promote good thermal contact with the plates to keep its temperature uniform. The length of sensing fiber under load was $50 \mathrm{~mm}$. At either end of the test length, the fiber was fixed in small rotation stages that could be rotated to vary the azimuthal angle of the applied transverse load. Peltier elements were used to keep the fiber at a fixed temperature. Before applying any load, the relative position of the cores was found by illuminating each core with $633 \mathrm{~nm}$ laser light and observing with a microscope the light scattered by the core. After setting the angle and focusing the microscope it was possible to infer the fiber orientation with a resolution of $\pm 5^{\circ}$.

\section{Results}

Figure 4 shows the reflected spectra of two of the four FBGs written in the MCF, under transverse loads of 0,100 , and $200 \mathrm{~N}$. Core 1 was located to one side of the fiber, with core 2 directly on the load axis. The spectra show a broadening and eventual splitting of the grating reflection peaks caused by the birefringence induced by the transverse strain. The structure in the no-load spectra is attributed to the grating 


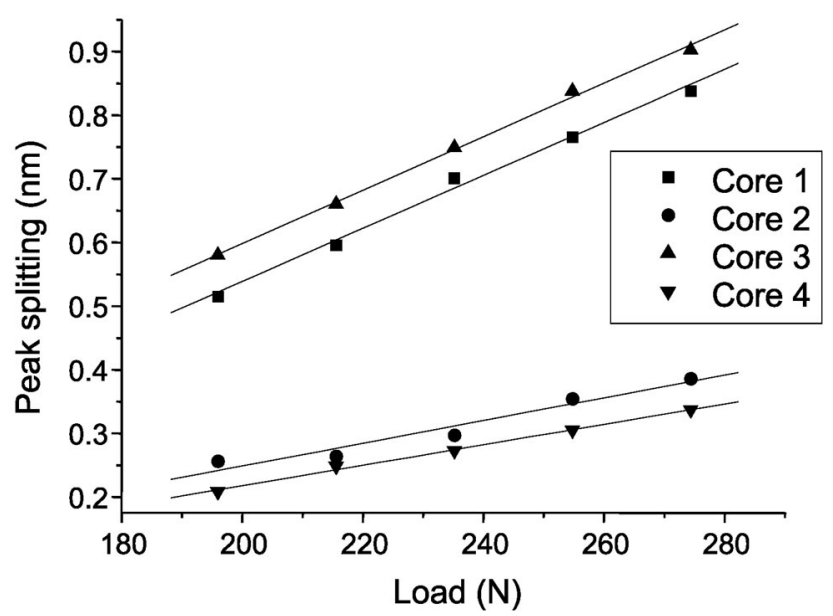

Fig. 6. Measurements of peak splitting versus load at $\theta=15^{\circ}$.

writing process, which was not sufficiently controlled to yield an ideal profile. The sidelobes around the second Bragg peak are likely due to inhomogeneities in the distribution of the loading force along the length of the fiber. The mean grating wavelength was taken to be the wavelength of maximum reflected power. Although there are more robust ways to define the grating wavelength, such as peak fitting or centroid calculation, the maximum power technique was deemed sufficient for this measurement. It is clear from Fig. 4 that the birefringence increases with load, and previous measurements ${ }^{8}$ have shown a linear relationship up to the maximum measured loading of $250 \mathrm{~N}$, or $2500 \mathrm{~N} \mathrm{~m}^{-1}$. The maximum sensitivity of the peak splitting was $0.44 \mathrm{pm}\left(\mathrm{N} \mathrm{m}^{-1}\right)^{-1}$, a value approximately three times that of FBGs in conventional single-core hi-bi fiber. ${ }^{5,6}$ The peak splittings in cores 1-4 are linear functions of load, as shown in Fig. 6. In this example the orientation angle $(\theta)$ of the fiber was such that the line formed by core 1 and 3 was $15^{\circ}$ from the load axis. The maximum measured load $(275 \mathrm{~N})$ corresponds to the limits of the loading rig and is not a physical limit. The minimum load corresponds to the minimum peak separation that could be resolved $(0.2 \mathrm{~nm}$ at $195 \mathrm{~N}$ in this particular example for cores 2 and 4). Modifying the experimental arrangement, for example, by separating the orthogonally polarized light components corresponding to each peak would enable smaller peak separations to be measured. The peak splitting is insensitive to common-mode effects such as axial strain and temperature, which to first order will shift the peak wavelengths of the FBGs in all the cores equally.

We investigated the angular dependence of the birefringence by changing the orientation of the fiber with respect to the load axis. The geometry is shown schematically in Fig. 7. The maximum birefringence corresponded to those cores located on the $y$ axis (the load axis) at $\theta=0$ and was a minimum in the cores at $\theta=90^{\circ}$. The FBG spectra in cores 3 and 4 were equivalent to the spectra in cores 1 and 2 , respectively, showing the symmetry of the stress field about

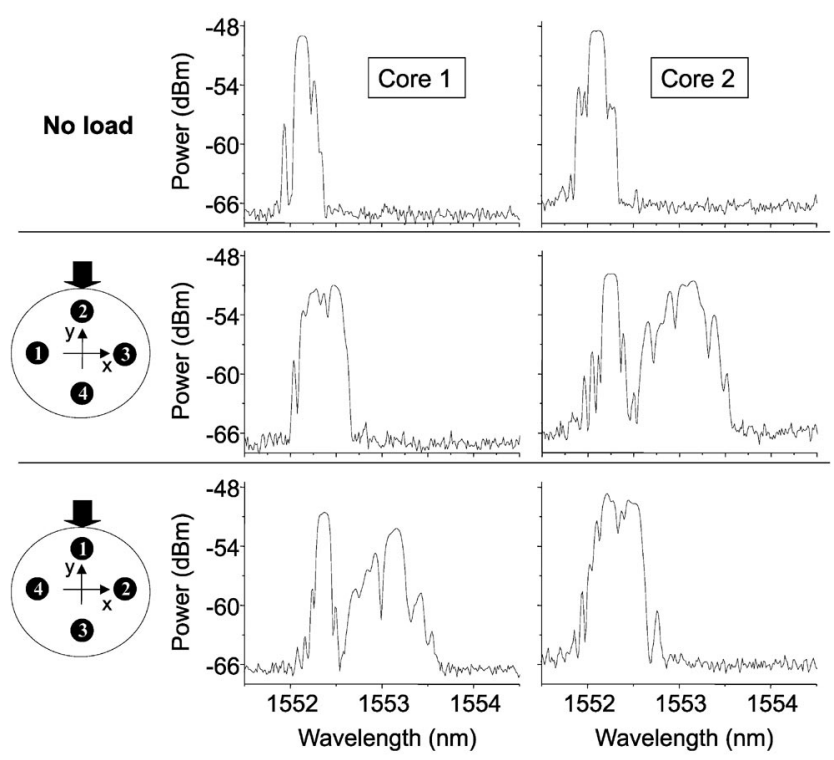

Fig. 7. FBG spectra for two orientations of MCF under a transverse load of $205 \mathrm{~N}$ compared with zero load (top).

the load axis. This symmetric behavior can also be seen in Fig. 6.

Figure 6 illustrates measurements from two neighboring cores for $\theta=15^{\circ}$. The splitting of each Bragg peak was recorded simultaneously as the load was increased, and the ratio of both slopes was $R$ $=2.73$, where $R$ is defined by

$$
R=\left(\frac{\mathrm{d} \Delta \lambda}{\mathrm{d} F}\right)_{i} /\left(\frac{\mathrm{d} \Delta \lambda}{\mathrm{d} F}\right)_{j}
$$

where $\Delta \lambda$ is the peak splitting, $F$ is the applied load, and $i$ and $j$ label the neighboring cores. Note that $R$ is the ratio of the slopes of the linear fits to the experimental points, which in practice are offset by an amount corresponding to the load threshold required to overcome slight nonuniformities in the loadbearing surfaces.

Using the theoretical model described in Section 4, the orientation of the MCF was evaluated from this particular ratio to be $\theta=16^{\circ}$ or $\theta=164^{\circ}$. The ambiguity arises from the symmetry of the function $R(\theta)$ about $\theta=90^{\circ}$, and it is not possible to discriminate the two solutions $\left(\theta\right.$ and $\left.180^{\circ}-\theta\right)$ that arise from a given ratio $R$. Experimental values of the ratio $R$ were obtained every $15^{\circ}$ using this technique and are shown in Fig. 8. Points taken in the second, third, and fourth quadrants have been plotted in the first quadrant by virtue of the MCF symmetry. The scatter of points is in part due to the error in measuring the real angle of rotation of the fiber. Nevertheless it can be seen that the experimental points confirm the theoretical prediction.

The resolution of the angle recovered from the experimental data was estimated from the plot of recovered angle versus set angle (Fig. 9). The scatter results in an angular resolution of $\pm 5^{\circ}$, as can be seen in the residuals of Fig. 9. 


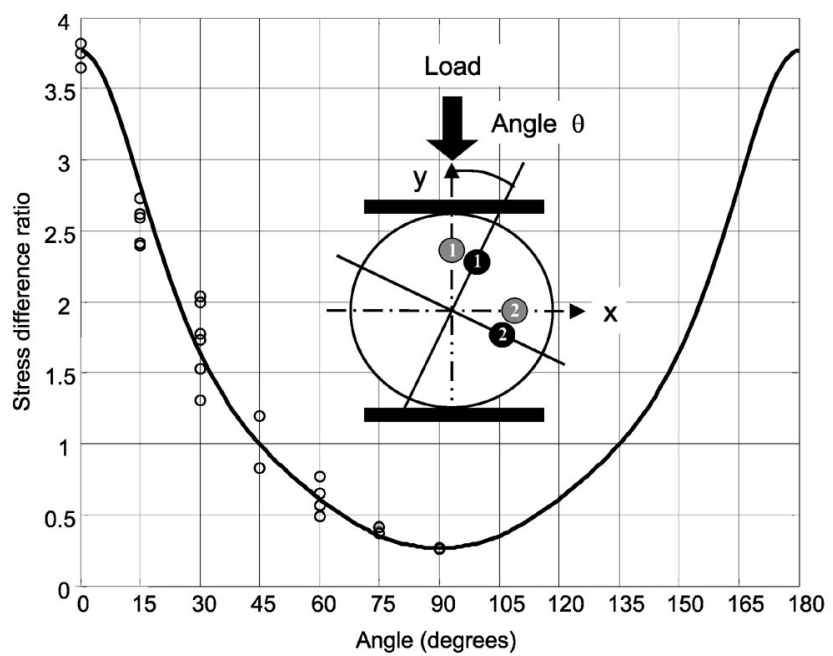

Fig. 8. Theoretical curve (solid curve) and experimental data (points) for the stress difference ratio of two cores at $90^{\circ}$ separation.

\section{Discussion}

\section{A. Comparison with Theoretical Stress Field}

The stresses through the cross section of a cylinder compressed between two diametrically opposite flat plates are well known from the theory of elasticity. ${ }^{12}$ This analytical model was used as an approximation for the MCF under load to calculate the stress field. The result is shown in Fig. 10, where the contours, or isochromatics, are plotted as a function of $(x, y)$ for the fiber cross section. These contours are the loci of the points where the difference between the rectangular stress components $\left(\sigma_{x}-\sigma_{y}\right)$ is constant. As expected, this vectorial stress field is symmetrical about the $y$ axis (the load axis) and the transverse strain is greatest close to the edge of the cladding on the diameter joining the points of load application. The birefringence at any point is proportional to the stress difference, ${ }^{2}$ i.e., to $\left(\sigma_{x}-\sigma_{y}\right)$; since different cores sample the stress field at different locations, the measured birefringence is a function of the orientation of the

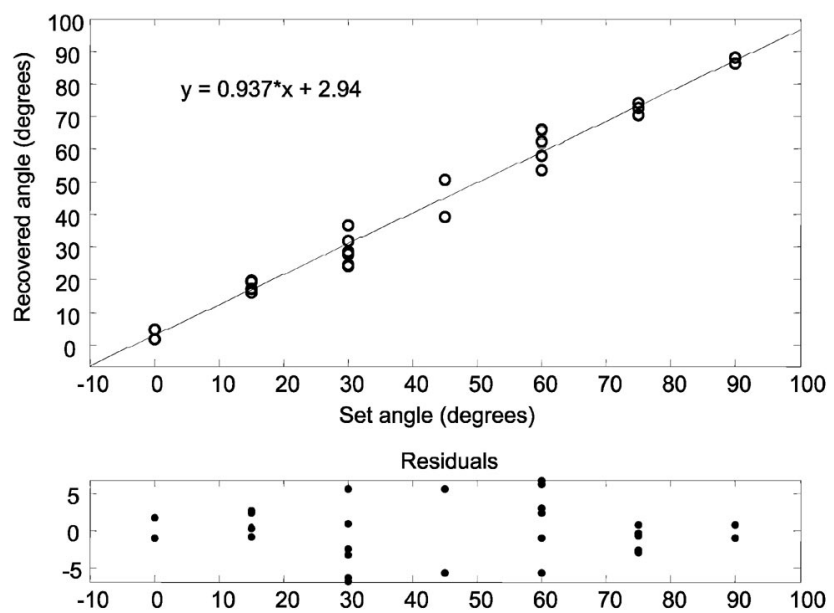

Fig. 9. Top, experimentally recovered angle of load axis versus the set angle. Bottom, residuals of the linear fit.

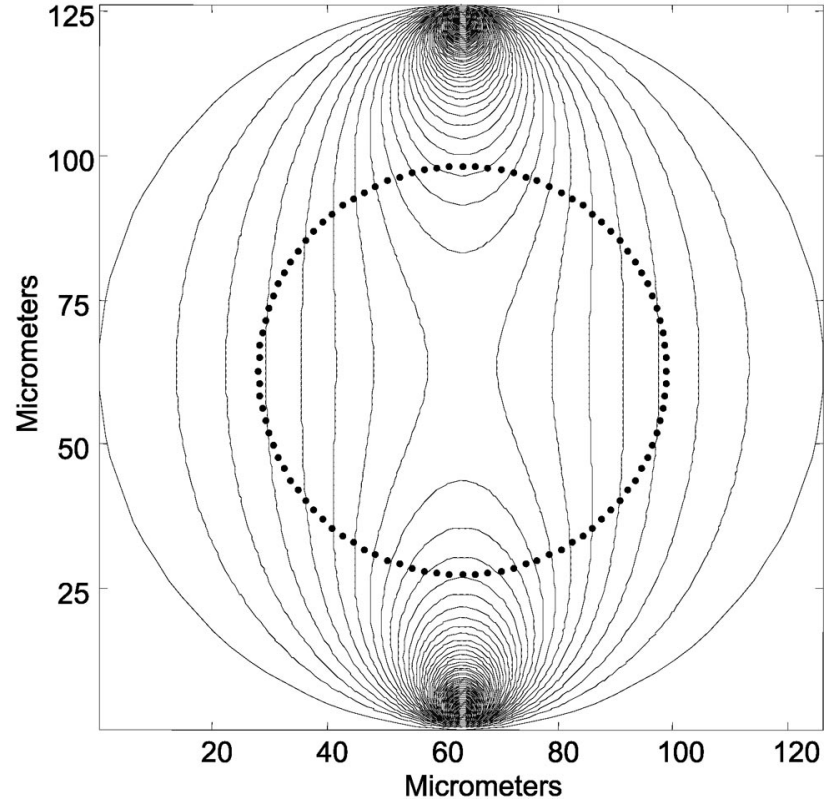

Fig. 10. Theoretical stress pattern of the MCF compressed by two diametrically opposite flat plates. The dotted circle shows the loci of points at which the cores sample the stress field.

applied load relative to the cores in addition to its magnitude. This model was also checked using a finite-element model software (LUSAS). By modeling a diametrally compressed cylinder we observed similar isochromatics.

It is interesting to note that the horizontal normal stress $\sigma_{x}$ is always positive or tensile in the whole cross section and the vertical normal stress $\sigma_{y}$ is negative or compressive (see Fig. 11). The refractiveindex changes in the cross section due to the photoelastic effect can be evaluated by ${ }^{13}$
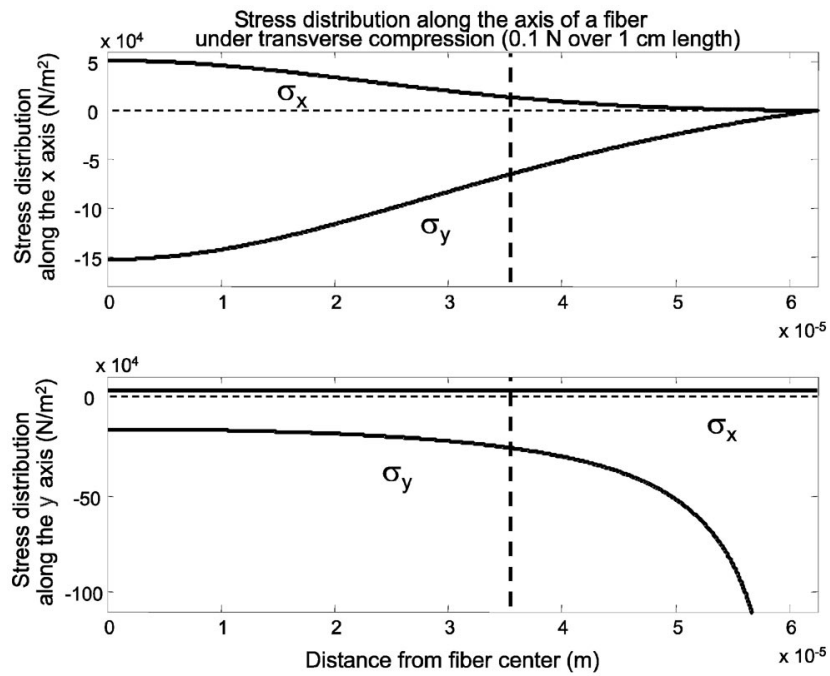

Fig. 11. Throughout the fiber cross section the horizontal norma stress $\sigma_{x}$ is always positive or tensile and the vertical normal stress $\sigma_{y}$ is negative or compressive. The dashed vertical lines indicate the radial location of the cores. 


$$
\begin{aligned}
n_{x}-n_{y} & =-\frac{n^{3}}{2 E}\left[p_{11}-p_{12}+v\left(p_{11}-p_{12}\right)\right]\left(\sigma_{x}-\sigma_{y}\right) \\
& =C\left(\sigma_{x}-\sigma_{y}\right),
\end{aligned}
$$

where $n$ is the effective refractive index of the fiber core, $E$ is Young's modulus, $p_{i j}$ are the Pockels coefficients, and $C$ is the stress-optic coefficient for silica fiber. As noted before, $\left(\sigma_{x}-\sigma_{y}\right)>0$, and since $p_{12}$ $>p_{11}$ for silica, then $n_{x}>n_{y}$. Therefore, according to Bragg's law, the difference between the Bragg wavelengths associated with each refractive index is $\lambda_{x}$ $-\lambda_{y}=2 \Lambda\left(n_{x}-n_{y}\right)$, where $\Lambda$ is the pitch of the FBG, and each Bragg peak belongs to a different orthogonal polarization state. We have previously shown experimentally this polarization dependence of the Bragg peaks in a transversely loaded MCF. ${ }^{8}$

Considering any two neighboring cores (1 and 2) with the geometric parameters of the MCF described above, and taking the ratio of the stress difference for these cores, we obtain

$$
\frac{\left(\sigma_{x}-\sigma_{y}\right)_{1}}{\left(\sigma_{x}-\sigma_{y}\right)_{2}}=\frac{\sigma_{x}(\theta)-\sigma_{y}(\theta)}{\sigma_{x}\left(\theta+\frac{\pi}{2}\right)-\sigma_{y}\left(\theta+\frac{\pi}{2}\right)} .
$$

By considering the splitting of the peak grating wavelengths for $x$ and $y$ polarizations $\left(\lambda_{x}-\lambda_{y}\right)$ to be proportional to these stresses, then it is possible to obtain the ratio from the peak splitting of the spectra, i.e.,

$$
R=\frac{\left(\lambda_{x}-\lambda_{y}\right)_{1}}{\left(\lambda_{x}-\lambda_{y}\right)_{2}}=\frac{\sigma_{x}(\theta)-\sigma_{y}(\theta)}{\sigma_{x}\left(\theta+\frac{\pi}{2}\right)-\sigma_{y}\left(\theta+\frac{\pi}{2}\right)} .
$$

The curve in Fig. 8 is a plot of this ratio as a function of the angle $\theta$, so that all possible positions of cores are covered. This theoretical analysis is in good agreement with experimental data, also shown in Fig. 8. The curve has a periodicity of $180^{\circ}$, and for the MCF used in the experiments it has maxima of 3.77 at $0^{\circ}$ and at $180^{\circ}$, corresponding to the positions where core 1 experiences maximum stress and core 2 experiences minimum stress. The ratio equals 1 when both cores are at $45^{\circ}$ from the vertical axis as might be expected from the symmetry of the stress field. It should be noted that, if we consider two opposite cores, $R=1$ irrespective of angle $\theta$ due to the symmetry of the stress field.

Consider Fig. 6 once more: As was explained above, the orientation was calculated to be $16^{\circ}$ or $164^{\circ}$ from the ratio of both slopes, with a resolution of $\pm 5^{\circ}$, i.e., core 1 is $16^{\circ}$ (or $164^{\circ}$ ) and core 2 is $106^{\circ}$ (or $74^{\circ}$ ) from the vertical axis. Once the positions of the cores are inferred, it is only necessary to use one of the cores to measure the applied load. The expression for the stress difference at any point $(x, y)$ within a cylindrical fiber compressed by two flat plates is

$$
\sigma_{x}-\sigma_{y}=\frac{F}{L} f(\theta)
$$

where $F / L$ is the force per unit length on the fiber and $f(\theta)$ is a function of the geometry of the fiber and the coordinates. By knowing the angle of one core, $f(\theta)$ can be solved. By combining Eqs. (2) and (5), it is possible to obtain the force per unit length on the sensing fiber as a function of the peak splitting $\Delta \lambda$ $=\left(\lambda_{x}-\lambda_{y}\right)$ observed in one core:

$$
\frac{F}{L}=\frac{f^{-1}(\theta)}{2 \Lambda C}\left(\lambda_{x}-\lambda_{y}\right)
$$

where $f^{-1}(\theta)$ works as a correction factor that depends on the location of the core from which we are measuring the peak splitting. Applying this technique to our data gives a load resolution of $\pm 15 \mathrm{~N} \mathrm{~m}^{-1}$ for loads up to $2500 \mathrm{~N} \mathrm{~m}^{-1}$. We believe that the resolution in the load can be improved significantly by use of a mechanically more efficient load transfer to the sensor fiber with a lower load threshold.

\section{B. Alternative Multicore Geometries for Improved Response}

Other MCFs can be proposed to reduce the uncertainty in the angular measurement and to increase the load sensitivity. The number of cores inside the cladding can be varied and is limited only by core-tocore coupling effects. To increase sensitivity, the cores can be placed closer to the edge of the fiber, resulting in a larger birefringence for a given load due to increased stress closer to the edge of the cladding. If the four-core MCF had the core separation increased from 50 to $72 \mu \mathrm{m}$ within a $125 \mu \mathrm{m}$ diameter cladding, the maximum peak splitting ratio $R$ would increase from 3.77 to 21.0 , a more than fivefold increase in load sensitivity.

To examine an improved angular response, we have modeled a three-core fiber with a standarddiameter $(125 \mu \mathrm{m})$ cladding and the cores at the same distance $(35.3 \mu \mathrm{m})$ from the fiber center as in the four-core fiber, but in this case they were separated by $120^{\circ}$. Using the above model, the stress difference ratio in a three-core fiber can be calculated as a function of angle and is plotted in Fig. 12 for the first two cores. The curve is no longer symmetric because the $120^{\circ}$ angular separation of the cores no longer coincides with the $90^{\circ}$ repeat of the stress field pattern. To check this prediction, experimental points were obtained indirectly from the four-core fiber results, as a three-core MCF was not available. Peak splitting measurements were taken from separate experiments to give data points for core pairs $120^{\circ}$ apart under the same transverse loading. As a result of our combining data from several experiments in this way, common-mode effects were not canceled; therefore the scatter in experimental points is somewhat larger than in previous tests. Nevertheless the combined experimental points follow the trend expected. 


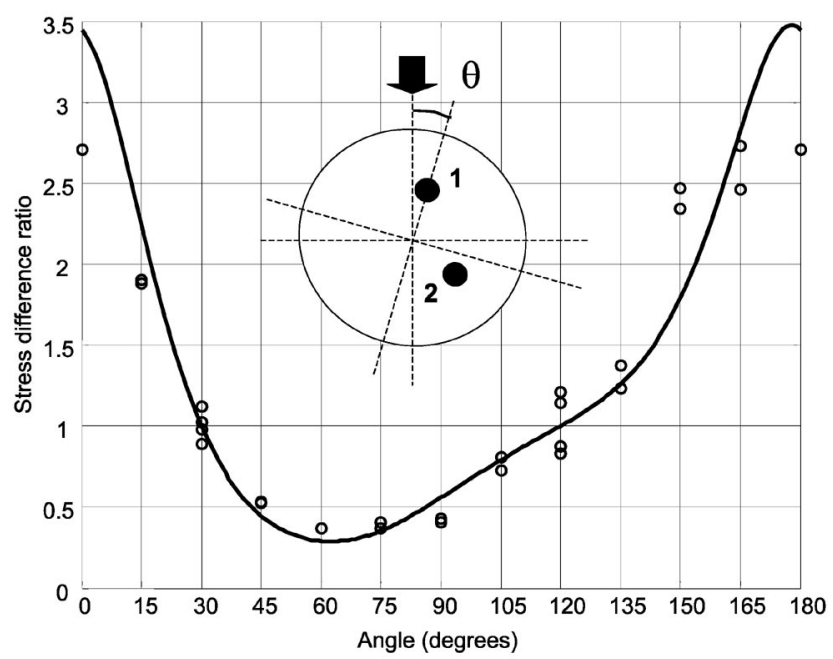

Fig. 12. Theoretical curve and experimental points of the stress difference ratio of the two first cores in a three-core fiber.

The stress difference ratios between all three possible core pairs are shown in Fig. 13. Although each curve is still double valued for a particular ratio, the load orientation angle can be found without ambiguity within the $180^{\circ}$ repeat period from any two pairs of cores. The method is demonstrated in the following example: Consider a ratio of 2.23 obtained from the birefringence of the core pair (1 and 2). According to the theoretical curve, the fiber can be rotated $15^{\circ}$ or $157^{\circ}$ from the vertical line to achieve a ratio of 2.23 . At the same time we can measure ratios of 2.83 and 1.27 from core pairs ( 1 and 3 ) and ( 2 and 3$)$, respectively. These ratios correspond to $15^{\circ}$ and $171^{\circ}$ in the former case and $15^{\circ}$ and $86^{\circ}$ in the latter. As we can see, the only consistent solution is $\theta=15^{\circ}$, thus eliminating the uncertainty in the angular position over a $180^{\circ}$ range.

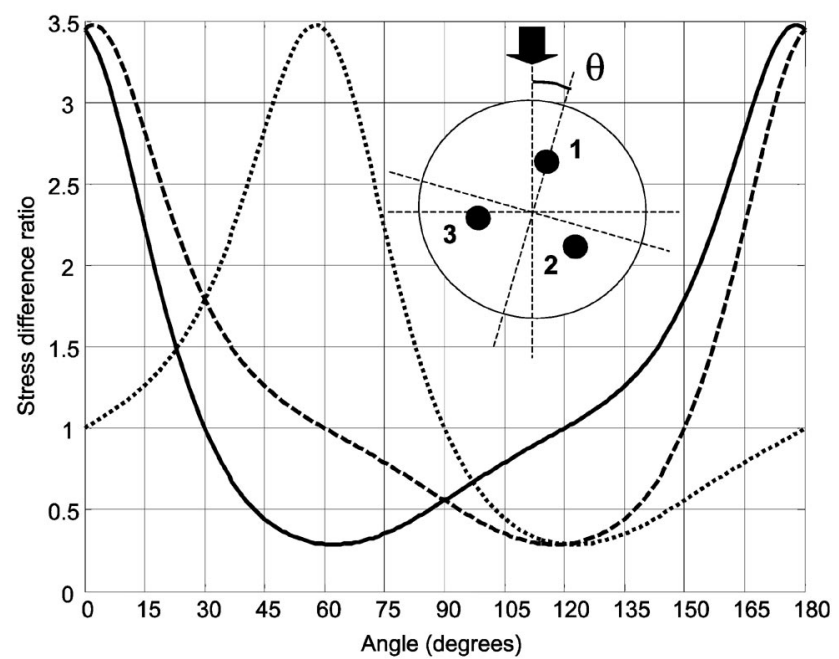

Fig. 13. Stress difference ratio simulated for the core pairs 1 and 2 (solid curve), 1 and 3 (dashed curve), 2 and 3 (dotted curve).

\section{Conclusions}

We have shown experimentally that Bragg gratings written in separate cores of a MCF can be used as sensing elements to measure the magnitude and direction of a transverse load applied along a fiber diameter at the location of the gratings. The Bragg peaks of individual cores are split by the birefringence induced by transverse loading. The load angle relative to the cores can be found from the ratio of the Bragg peak splitting between pairs of cores. The experimentally observed ratios of peak splitting were consistent with the analytical solution for the stress field of a diametrally compressed homogeneous cylinder. A four-core MCF allowed the angle of the load axis to be determined within a $90^{\circ}$ repeat period, while a three-core $\mathrm{MCF}$ (i.e., with $120^{\circ}$ core spacing) can determine the load axis within a $180^{\circ}$ ambiguity. An angular resolution of $5^{\circ}$ was obtained with a fourcore MCF.

At a given orientation, the peak splitting was linearly dependent on loading, and by determining the load angle from the peak splitting ratios, we could infer the load from the peak splitting to a resolution of $\pm 15 \mathrm{~N} \mathrm{~m}^{-1}$ over a load range up to $2500 \mathrm{~N} \mathrm{~m}^{-1}$. The sensitivity of the technique could be improved by use of a MCF with cores closer to the cladding boundary. The potential applications include measurement of internal transverse stresses in composite materials and compact load cells with a MCF supported within a V-groove mounting.

The authors acknowledge the UK Engineering and Physical Science Research Council (EPSRC) and UK Defence Science and Technology Laboratory for provision of funding through the Joint Grant Scheme. W. N. MacPherson and A. J. Moore acknowledge the EPSRC for provision of funding through the Advanced Fellowship Programme. The authors thank G. Fleming (NASA Langley) for supply of the multicore fiber.

\section{References}

1. P. D. Gianino and B. Bendow, "Calculations of stress-induced changes in the transverse refractive-index profile of optical fibers," Appl. Opt. 20, 430-434 (1981).

2. K. Okamoto, T. Hosaka, and T. Edahiro, "Stress analysis of optical fibers by a finite element method," IEEE J. Quantum Electron. QE-17, 2123-2129 (1981).

3. K. S. C. Kuang, R. Kenny, M. P. Whelan, W. J. Cantwell, and P. R. Chalker "Embedded fibre Bragg grating sensors in advanced composite materials," Compos. Sci. Technol. 61, 13791387 (2001).

4. J. A. Guemes and J. M. Menéndez, "Response of Bragg grating fiber-optic sensors when embedded in composite laminates," Compos. Sci. Technol. 62, 959-966 (2002).

5. C. M. Lawrence, D. V. Nelson, E. Udd, and T. Bennett, "A fiber optic sensor for transverse strain measurement," Exp. Mech. 39, 202-209 (1999).

6. I. Abe, H. J. Kalinowski, O. Frazão, J. L. Santos, R. N. Nogueira, and J. L. Pinto, "Superimposed Bragg gratings in high-birefringence fibre optics: three-parameter simultaneous measurements," Meas. Sci. Technol. 15, 1453-1457 (2004).

7. L. Zhang, Y. Liu, L. Everall, J. A. R. Williams, and I. Bennion, "Design and realization of long-period grating devices in con- 
ventional and high birefringence fibers and their novel applications as fiber-optic load sensors," IEEE J. Sel. Top. Quantum Electron. 5, 1373-1378 (1999).

8. M. Silva-Lopez, C. Li, W. N. MacPherson, A. J. Moore, J. S. Barton, J. D. C. Jones, D. Zhao, L. Zhang, and I. Bennion, "Differential birefringence in multicore fiber Bragg gratings under transverse stress," Opt. Lett. 29, 2225-2227 (2004).

9. B. Rosinski, J. W. D. Chi, P. Grosso, and J. Le Bihan, "Multichannel transmission of a multicore fiber coupled with vertical-cavity surface-emitting lasers," J. Lightwave Technol. 17, 807-810 (1999).
10. D. L. Williams, B. J. Ainslie, J. R. Armitage, R. Kashyap, and R. Campbell, "Enhanced UV photosensitivity in boron codoped germanosilicate fibres," Electron. Lett. 29, 45-47 (1993).

11. G. M. H. Flockhart, W. N. MacPherson, J. S. Barton, J. D. C. Jones, L. Zhang, and I. Bennion, "Two-axis bend measurement with Bragg gratings in multicore optical fiber," Opt. Lett. 28, 387-389 (2003).

12. M. Frocht, Photoelasticity (Wiley, 1948), Vol. 2.

13. R. Gafsi and M. A. El-Sherif, "Analysis of inducedbirefringence effects on fiber Bragg gratings," Opt. Fiber Technol. 6, 299-323 (2000). 